\title{
Distributed Finite-Time Nash Equilibrium Seeking for Non-Cooperative Games
}

\author{
Xiao Fang ${ }^{1}$, Jinhu Lü $^{2, *}$ and Guanghui Wen ${ }^{1}$ \\ 1 School of Mathematics, Southeast University, Nanjing 211189, P.R. China. \\ 2 School of Automation Science and Electrical Engineering, Beihang University, \\ Beijing 100083, P.R. China.
}

Received 29 June 2020; Accepted 28 December 2020

\begin{abstract}
The paper aims to design a distributed algorithm for players in games such that the players can learn Nash equilibriums of non-cooperative games in finite time. We first consider the quadratic non-cooperative games and design estimate protocols for the players such that they can estimate all the other players' actions in distributed manners. In order to make the players track all the other players' real actions in finite time, a bounded gradient dynamics is designed for players to update their actions by using the estimate information. Then the algorithm is extended to more general noncooperative games and it is proved that players' estimates can converge to all the other players' real actions in finite time and all players can learn the unique Nash equilibrium in finite time under mild assumptions. Finally, simulation examples are provided to verify the validity of the proposed finite-time distributed Nash equilibrium seeking algorithms.
\end{abstract}

AMS subject classifications: 91A43, 93C10, 93D99

Key words: Nash equilibrium, non-cooperative game, distributed algorithm, finite-time convergence.

\section{Introduction}

Non-cooperative game is an important branch of game in which the cost function of each player relies on its action and other players' actions and each player wants to minimize its cost function selfishly. Nash equilibrium seeking problem as one of research emphases of non-cooperative games, aims to find a strategy combination under which no player can minimize its cost function by changing its strategy individually [1].

\footnotetext{
*Corresponding author. Email addresses: fx_ysu@163.com (X. Fang), jhlu@iss.ac.cn (J. Lü), wenguanghui@gmail . com (G. Wen) 
In recent years, various distributed Nash equilibrium seeking algorithms have been proposed under the problem setting where the players only know part of the decision information (see [2-4] and references therein). The players have limited decision information in networked games while their cost functions depend on global decision information concluding all players' strategies. For non-cooperative games, global decision information is the prerequisite for each player to make the best response to other players' strategies. In the existing literature for non-cooperative games with partial-decision information setting, a common idea is to use the estimation of global decision information to update players' strategies. To be more specific, the networked players exchange their estimates of all other players' strategies based on the underlying communication graph. Then, the consensus idea is used to make the estimated information converge to the real global decision information, and the gradient strategy is used to make the actions of players converge to Nash equilibrium.

The main difference between distributed optimization and non-cooperative game lies in that agents aim to minimize a sum of their local objective functions cooperatively $[5,6]$ in distributed optimization while players want to minimize their own objective functions selfishly in non-cooperative games, i.e., the purpose of distributed optimization is to solve

$$
\min _{x} \sum_{i=1}^{N} f_{i}(x)
$$

while the purpose of players in non-cooperative games is

$$
\min _{x_{i}} f_{i}(x), \quad \forall i=1, \cdots, N
$$

At present, some literatures have studied the problem of finite-time optimization (e.g., [7-9] and references therein). Although some authors have studied the accelerated Nash equilibrium seeking algorithm, for example, [10] proposed an accelerated version of the gradient play algorithm with a faster convergence for Nash equilibrium seeking problem and [11] investigated Nash equilibrium seeking problem via alternating direction method of multipliers, these works still stay on the results of asymptotic convergence.

Motivated by finite-time distributed optimization, in this paper we aim to solve the finite-time Nash equilibrium seeking problem. In this paper, the players still keep estimates on all the other players' actions and update their actions by employing these estimates. Gradient play is still adopted here. Firstly, in order to make the estimates track players' real actions in finite time, we make some improvements on the basis of gradient play to ensure the update rate of players' actions is bounded. Secondly, we combine sign function and the idea of consensus to design update algorithm for players' estimates. The analysis of finite-time Nash equilibrium seeking is carried out in two stages: (1) the estimates converge to players' actions in limited time under the assumption of connected communication graph; (2) all players' actions converge to Nash equilibrium in finite time after the first stage under some assumptions. 
The rest of this paper is organized as follows. Some basic notations are given and non-cooperative game model and basic assumptions are introduced in Section 2. Section 3 proposes a finite-time distributed Nash equilibrium seeking algorithm for quadratic games and proves that the estimates converge to players' actions in limited time and all players learn the Nash equilibrium in finite time. In Section 4, more general noncooperative games are considered and the finite-time Nash equilibrium seeking problem is solved under the proposed distributed algorithm. Section 5 gives some examples to illustrate that these two proposed algorithms are valid. Section 6 concludes this paper.

\section{Preliminaries and problem formulation}

\subsection{Graph theory and basic notations}

Let $\mathbb{R}^{n}$ and $\mathbb{R}^{m \times n}$ be $n$-dimensional column vectors space and $m \times n$-dimensional matrices space, respectively. $x^{T}$ and $A^{T}$ denote the transpose of vector $x \in \mathbb{R}^{n}$ and matrix $A \in \mathbb{R}^{m \times n}$. $\|\cdot\|_{1}$ and $\|\cdot\|_{2}$ represent 1-norm and 2-norm of vectors or matrices, respectively. For $x \in \mathbb{R}^{n},\|x\|_{2} \leq\|x\|_{1}$. For a real symmetrical matrix $A, \rho(A)=\|A\|_{2}$ where $\rho(A)$ is the spectral radius of matrix $A$. Let $\lambda_{\min }(A)$ and $\lambda_{\max }(A)$ denote respectively the minimum and maximum eigenvalues of matrix $A . \operatorname{sign}(\cdot)$ is the standard sign function. $1_{n}$ and $0_{n}$ are $n$-dimensional column vectors with all elements equal 1 and 0 , respectively. $I_{N}$ is $N$-dimensional identity matrix. $\otimes$ is Kronecker product. Sometimes, a vector $x$ is also represented by $\left[x_{i}\right]_{\mathrm{vec}}$ with $x_{i}$ being its component.

An undirected graph can be modelled as $\mathcal{G}=(V, E)$ with nodes set $V=\{1, \cdots, N\}$ and edges set $E \subseteq V \times V$. The adjacency matrix of $\mathcal{G}$ is defined as $A=\left[a_{i j}\right]_{N \times N}$ with $a_{i j}=a_{j i}>0$ if there is an edge between nodes $i$ and $j$, i.e., $i$ and $j$ can deliver information with each other directly and they are neighbors with each other. $a_{i j}=a_{j i}=0$, otherwise. The Laplacian matrix of the adjacency matrix $A$ is defined as $L=\left[l_{i j}\right]_{N \times N}$ with $l_{i i}=\sum_{i=1}^{N} a_{i j}$ and $l_{i j}=-a_{i j}$ for $i \neq j . \mathcal{G}$ is called connected if there is path between any two distinct nodes $i, j \in V$. For undirected and connected graph $\mathcal{G}$, the Laplacian matrix $L$ has a simple zero eigenvalue with $1_{N}$ being its eigenvector and the other eigenvalues are positive.

\subsection{Problem formulation}

Consider $N$ players in a non-cooperative game with $x_{i} \in \mathbb{R}^{n_{i}}$ being player $i^{\prime}$ s action. Let $\mathcal{N}=\{1, \cdots, N\}$ denote the set of players and $x=\left[x_{1}^{T}, \cdots, x_{N}^{T}\right]^{T}$ be a strategy combination of all players' actions. The cost function of player $i$ is represented by $f_{i}(x)$ that is a function of player $i^{\prime}$ s action and other players' actions. Let $x_{-i}=\left[x_{1}^{T}, \cdots, x_{i-1}^{T}, x_{i+1}^{T}, \cdots, x_{N}^{T}\right]^{T}$ is a vector consisting of all the other players' actions except $x_{i}$. In the absence of ambiguity, $x=\left(x_{i}, x_{-i}\right)$ and $f_{i}(x)$ can also be written as $f_{i}\left(x_{i}, x_{-i}\right)$ in this paper. All players have the intention to minimize their cost functions selfishly. Nash equilibrium is a special strategy combination under which each player will pay more cost if it changes its strategy 
individually. A rigorous mathematical definition of a Nash equilibrium is given in the following.

Definition 2.1. $x^{*}=\left(x_{i}^{*}, x_{-i}^{*}\right)$ is a Nash equilibrium if and only if the following holds,

$$
f_{i}\left(x_{i}^{*}, x_{-i}^{*}\right) \leq f_{i}\left(x_{i}, x_{-i}^{*}\right), \quad \forall i \in \mathcal{N} .
$$

Remark 2.1. According to the definition of Nash equilibrium, one knows that $\frac{\partial f_{i}\left(x^{*}\right)}{\partial x_{i}}=0$ for any $i \in \mathcal{N}$.

In the problem setting, however, the players have no access to non-neighboring players' actions. Player $i$ needs to achieve the information about $x_{-i}$ or estimate information of $x_{-i}$ before changing its own action to minimize it cost. Let $y_{i j}$ be player $i$ 's estimate on $x_{j}$ and $y_{i}=\left[y_{i 1}^{T}, \cdots, y_{i N}^{T}\right]^{T}$ denote player $i$ 's estimate on $x$. The players can exchange estimate information with their neighbors to track the real actions of other players and the communication topology of players is represented by undirected graph $\mathcal{G}$. The players update their actions by using their estimates $y_{i}, i \in \mathcal{N}$. Our purpose is to design a distributed Nash equilibrium seeking algorithm such that the players learn the Nash equilibrium in finite time by using only local information.

In order to ensure the existence of Nash equilibrium, the sufficient existence assumption is given.

Assumption 2.1. $f_{i}(x)$ is convex on $x_{i}$ for given $x_{-i}$ and $f_{i}(x)$ is twice continuous differentiable with $x$ for any $i \in \mathcal{N}$.

In order to ensure each player's action can be tracked by all the other players, the following assumption is also needed.

Assumption 2.2. The communication topology $\mathcal{G}$ is undirected and connected.

Remark 2.2. Assumptions 2.1 and 2.2 are a common set of assumptions in the study of non-cooperative game over networks (see, e.g. [3,4] and reference therein). Assumption 2.1 is easily satisfied in some scenarios, such as the coordination problems in mobile sensor networks [14] and the energy consumption control of the plug-in hybrid electric vehicles [15].

\section{Distributed finite-time Nash equilibrium seeking for quadratic non-cooperative games}

In this section, we consider Nash equilibrium seeking problem for a simple quadratic non-cooperative game and propose a distributed algorithm for players such that their estimates can track all the other players' real actions in finite time and the players can also learn the Nash equilibrium in finite time. 
For quadratic games, players' cost functions can be represented as

$$
f_{i}(x)=\frac{1}{2} \sum_{j=1}^{N} \sum_{k=1}^{N} x_{j}^{T} H_{j k}^{i} x_{k}+\sum_{j=1}^{N} h_{j}^{i^{T}} x_{j}+v_{i},
$$

where $H_{j k}^{i} \in \mathbb{R}^{n_{j} \times n_{k}}$ is constant matrix satisfying $H_{j k}^{i}{ }^{T}=H_{k j}^{i}$, and $h_{j}^{i} \in \mathbb{R}^{n_{j}}$ and $v_{i} \in \mathbb{R}^{n_{i}}$ are constant vectors for $i \in \mathcal{N}$.

Let $\Gamma(x)=\left[\begin{array}{llll}{\frac{\partial f_{1}(x)}{\partial x_{1}}}^{T} & \frac{\partial f_{2}(x)}{\partial x_{2}} & \cdots & {\frac{\partial f_{N}(x)}{\partial x_{N}}}^{T}\end{array}\right]^{T}$, then we have $\Gamma(x)=H x+h$ where

$$
H=\left[\begin{array}{cccc}
H_{11}^{1} & H_{12}^{1} & \cdots & H_{1 N}^{1} \\
H_{21}^{2} & H_{22}^{2} & \cdots & H_{2 N}^{2} \\
\vdots & \vdots & \ddots & \vdots \\
H_{N 1}^{N} & H_{N 2}^{N} & \cdots & H_{N N}^{N}
\end{array}\right], \quad h=\left[\begin{array}{llll}
h_{1}^{1^{T}} & h_{2}^{2^{T}} & \cdots & h_{N}^{N^{T}}
\end{array}\right]^{T} .
$$

Let $\Gamma(x)=0$, if $H$ is invertible, we can get the unique Nash equilibrium $x^{*}=-H^{-1} h$ by direct calculation [1, Proposition 4.5].

Assumption 3.1. $H_{i i}^{i}>0, H_{i j}^{i}=H_{j i}^{j}$ and $H$ is strictly diagonally dominant.

Remark 3.1. Note that a game satisfying Assumption 3.1 can be seen as a potential game [16] with the potential function being strictly convex about $x$. Assumption 3.1 ensures the uniqueness of Nash equilibrium and $H$ is a symmetric positive definite matrix.

The distributed Nash equilibrium seeking algorithm for player $i, i \in \mathcal{N}$ is designed as follows:

$$
\begin{aligned}
& \dot{x}_{i}=-\alpha_{i} \frac{\frac{\partial f_{i}\left(y_{i}\right)}{\partial x_{i}}}{\left\|\frac{\partial f_{i}\left(y_{i}\right)}{\partial x_{i}}\right\|_{2}}, \\
& \dot{y}_{i j}=-\delta_{i} \operatorname{sign}\left(\sum_{k=1}^{N} a_{i k}\left(y_{i j}-y_{k j}\right)+a_{i j}\left(y_{i j}-x_{j}\right)\right),
\end{aligned}
$$

where $\alpha_{i}>0, \delta_{i}>0$ and $\frac{\partial f_{i}\left(y_{i}\right)}{\partial x_{i}}=\left.\frac{\partial f_{i}(x)}{\partial x_{i}}\right|_{x=y_{i}}$ and $a_{i k}$ is $(i, k)$-th entry of the adjacency matrix $A$ corresponding to communication topology $\mathcal{G}$. It should be noticed that for a vector $x \in \mathbb{R}^{n}$, define $\frac{x}{\|x\|_{2}}=0_{n}$ if $x=0_{n}$.

From Eq. (3.3), we know that $\dot{x}_{i}$ is bounded and $\left\|\dot{x}_{i}\right\|_{2}=\alpha_{i}$. Furthermore, $\|\dot{x}\|_{2} \leq$ $\sum_{i=1}^{N}\left\|\dot{x}_{i}\right\|_{2}=\sum_{i=1}^{N} \alpha_{i}$ where $x=\left[x_{1}^{T}, \cdots, x_{N}^{T}\right]^{T}$. In order to simplify analysis, we assume that $x_{i} \in \mathbb{R}, \forall i \in \mathcal{N}$ in the rest of this paper. For $x_{i} \in \mathbb{R}^{n_{i}}\left(n_{i}>1\right)$, the analysis is similar.

Theorem 3.1. If the undirected graph $\mathcal{G}$ is connected and there is a constant $\mu>0$ such that $\|\dot{x}\|_{2} \leq \mu$, then for any $i, j \in \mathcal{N}$, the estimate $y_{i j}$ can converge to $x_{j}$ in finite time by choosing $\delta_{i}$ satisfying $\min _{i \in \mathcal{N}}\left\{\delta_{i}\right\}>\sqrt{N} \mu$. 
Proof. It follows from the definition of the Laplacian matrix that $L 1_{N}=0_{N}$. Let $y=$ $\left[\begin{array}{llll}y_{1}^{T} & y_{2}^{T} & \cdots & y_{N}^{T}\end{array}\right]^{T}$, then the estimate dynamics of players in compactness form can be written as

$$
\dot{y}=-\delta \operatorname{sign}\left(\left(L \otimes I_{N}+B\right)\left(y-1_{N} \otimes x\right)\right),
$$

where $\delta=\operatorname{diag}\left\{\delta_{i}\right\} \otimes I_{N}, i=1, \cdots, N$ and $B=\operatorname{diag}\left\{\left[a_{11}, \cdots, a_{1 N}, \cdots, a_{N 1}, \cdots, a_{N N}\right]\right\}$.

Since $\mathcal{G}$ is undirected and connected, then 0 is a simple eigenvalue of the Laplacian matrix $L$, according to Gershgorin's disc theorem, $L \otimes I_{N}+B$ is symmetric and positive definite. Construct the candidate Lyapunov function as

$$
V=\frac{1}{2}\left(y-1_{N} \otimes x\right)^{T}\left(L \otimes I_{N}+B\right)\left(y-1_{N} \otimes x\right) .
$$

Then we have

$$
\frac{1}{2} \lambda_{\min }\left(L \otimes I_{N}+B\right)\left\|y-1_{N} \otimes x\right\|_{2}^{2} \leq V \leq \frac{1}{2} \lambda_{\max }\left(L \otimes I_{N}+B\right)\left\|y-1_{N} \otimes x\right\|_{2}^{2} .
$$

Taking the derivative of $V$ with respect to time $t$,

$$
\begin{aligned}
\dot{V} & =\left(y-1_{N} \otimes x\right)^{T}\left(L \otimes I_{N}+B\right)\left[-\delta \operatorname{sign}\left(\left(L \otimes I_{N}+B\right)\left(y-1_{N} \otimes x\right)\right)-1_{N} \otimes \dot{x}\right] \\
& \leq-\min _{i \in \mathcal{N}}\left\{\delta_{i}\right\}\left\|\left(L \otimes I_{N}+B\right)\left(y-1_{N} \otimes x\right)\right\|_{1}+\left\|\left(L \otimes I_{N}+B\right)\left(y-1_{N} \otimes x\right)\right\|_{2}\left\|1_{N} \otimes \dot{x}\right\|_{2} \\
& \leq-\min _{i \in \mathcal{N}}\left\{\delta_{i}\right\}\left\|\left(L \otimes I_{N}+B\right)\left(y-1_{N} \otimes x\right)\right\|_{2}+\left\|\left(L \otimes I_{N}+B\right)\left(y-1_{N} \otimes x\right)\right\|_{2}\left\|1_{N} \otimes \dot{x}\right\|_{2} \\
& \leq-\left(\min _{i \in \mathcal{N}}\left\{\delta_{i}\right\}-\sqrt{N} \mu\right)\left\|\left(L \otimes I_{N}+B\right)\left(y-1_{N} \otimes x\right)\right\|_{2} \\
& \leq-\left(\min _{i \in \mathcal{N}}\left\{\delta_{i}\right\}-\sqrt{N} \mu\right) \lambda_{\min }\left(L \otimes I_{N}+B\right)\left\|\left(y-1_{N} \otimes x\right)\right\|_{2} \\
& \leq-\left(\min _{i \in \mathcal{N}}\left\{\delta_{i}\right\}-\sqrt{N} \mu\right) \lambda_{\min }\left(L \otimes I_{N}+B\right) \sqrt{\frac{2 V}{\lambda_{\max }\left(L \otimes I_{N}+B\right)}} .
\end{aligned}
$$

Since $\min _{i \in \mathcal{N}}\left\{\delta_{i}\right\}>\sqrt{N} \mu$, it follows that $\dot{V} \leq 0$ and the equation is satisfied if and only if $V=0$. Further,

$$
\frac{\dot{V}}{\sqrt{V}} \leq-\left(\min _{i \in \mathcal{N}}\left\{\delta_{i}\right\}-\sqrt{N} \mu\right) \sqrt{\frac{2 \lambda_{\min }^{2}\left(L \otimes I_{N}+B\right)}{\lambda_{\max }\left(L \otimes I_{N}+B\right)}} .
$$

Integrating both sides of Eq. (3.8) from $t_{0}=0$ to $t$, we have

$$
2 \sqrt{V(t)}-2 \sqrt{V(0)} \leq-\left(\min _{i \in \mathcal{N}}\left\{\delta_{i}\right\}-\sqrt{N} \mu\right) \sqrt{\frac{2 \lambda_{\min }^{2}\left(L \otimes I_{N}+B\right)}{\lambda_{\max }\left(L \otimes I_{N}+B\right)}} t .
$$

Therefore, for any $i, j \in \mathcal{N}$, the estimate information $y_{i j}$ converges to $x_{j}$ in finite time

$$
\mathrm{T}_{1} \leq \frac{\sqrt{V(0)}}{\left(\min \left\{\delta_{i}\right\}-\sqrt{N} \mu\right)} \times \sqrt{\frac{2 \lambda_{\max }\left(L \otimes I_{N}+B\right)}{\lambda_{\min }^{2}\left(L \otimes I_{N}+B\right)}} .
$$

In other words, $y_{i j}=x_{j}$ for $t \geq T_{1}$. 
Remark 3.2. Note that $\min _{i \in \mathcal{N}}\left\{\delta_{i}\right\}>\sqrt{N} \mu$ is a sufficient condition but not necessary condition in Theorem 3.1. Moreover, it follows from the expression of the upper bound of $T_{1}$ that for given $N$ and $\mu, T_{1}$ can be small enough by choosing $\min _{i \in \mathcal{N}}\left\{\delta_{i}\right\}$ large enough.

On the basis of Theorem 3.1, we have the following Nash equilibrium convergence theorem for finite time.

Theorem 3.2. Suppose that Assumptions 2.1-3.1 are satisfied. Then the players' actions can converge to the unique Nash equilibrium for quadratic non-cooperative game under distributed algorithm (3.3).

Proof. It follows from Theorem 3.1 that $y_{i j}$ can converge to $x_{j}$ in finite time $T_{1}$ by choosing appropriate $\delta_{i}$, which means that $y_{i}=x$ for any $i \in \mathcal{N}$ when $t \geq T_{1}$. Therefore, for $\forall t \geq T_{1}$, the dynamics of players' actions in Eq. (3.3) becomes

$$
\dot{x}_{i}=-\alpha_{i} \frac{\frac{\partial f_{i}(x)}{\partial x_{i}}}{\left\|\frac{\partial f_{i}(x)}{\partial x_{i}}\right\|_{2}}, \quad \forall i \in \mathcal{N} \text {. }
$$

Let

$$
\hat{\Gamma}(x)=\left[\begin{array}{llll}
\frac{\frac{\partial f_{1}(x)^{T}}{\partial x_{1}}}{\left\|\frac{\partial f_{1}(x)}{\partial x_{1}}\right\|_{2}} & \frac{\frac{\partial f_{2}(x)^{T}}{\partial x_{2}}}{\left\|\frac{\partial f_{2}(x)}{\partial x_{2}}\right\|_{2}} & \cdots & \frac{\frac{\partial f_{N}(x)^{T}}{\partial x_{N}}}{\left\|\frac{\partial f_{N}(x)}{\partial x_{N}}\right\|_{2}}
\end{array}\right]^{T},
$$

then the compactness form of the dynamics of players' actions can be written as

$$
\dot{x}(t)=-\alpha \hat{\Gamma}(x), \quad t \geq T_{1},
$$

where $\alpha=\operatorname{diag}\left\{\alpha_{i}\right\}$ is a positive definite matrix due to $\alpha_{i}>0, \forall i \in \mathcal{N}$.

According to Assumption 3.1, matrix $H$ is symmetric and positive definite. In order to analyze the stability of Nash equilibrium, the following candidate Lyapunov function is constructed,

$$
V_{1}=\frac{1}{2} \Gamma(x)^{T} \alpha^{-1} H^{-1} \Gamma(x) .
$$

The Lyapunov function is reasonably constructed because $\Gamma(x)=H x+h=0_{N}$ holds if and only if $x=x^{*}$ where $x^{*}$ is the unique Nash equilibrium. Take the derivative of $\Gamma(x)$ with respect to $t$, then $\dot{\Gamma}(x)=-H \alpha \hat{\Gamma}(x)$.

Therefore, for $t \geq T_{1}$, we can get that

$$
\begin{aligned}
\dot{V}_{1} & =\Gamma(x)^{T} \alpha^{-1} H^{-1}(-H \alpha \hat{\Gamma}(x)) \\
& =-\Gamma(x)^{T} \hat{\Gamma}(x) \\
& =-\sum_{i=1}^{N}\left\|\frac{\partial f_{i}(x)}{\partial x_{i}}\right\|_{2} \\
& \leq-\|\Gamma(x)\|_{2} \\
& \leq-\sqrt{\frac{2 V_{1}}{\lambda_{\max }\left(\alpha^{-1} H^{-1}\right)}}
\end{aligned}
$$


Similarly, one has

$$
2 \sqrt{V_{1}(t)}-2 \sqrt{V_{1}\left(T_{1}\right)} \leq-\sqrt{\frac{2}{\lambda_{\max }\left(\alpha^{-1} H^{-1}\right)}}\left(t-T_{1}\right),
$$

which indicates that there is a finite time $T_{2} \leq \sqrt{2 V_{1}\left(T_{1}\right) \lambda_{\max }\left(\alpha^{-1} H^{-1}\right)}$ such that $x=x^{*}$ for $t \geq T_{1}+T_{2}$.

\section{Distributed finite-time Nash equilibrium seeking for general non-cooperative games}

In this section, we will consider the finite-time Nash equilibrium seeking problem for more general non-cooperative games. Suppose that there are $N$ players and their cost functions satisfy Assumption 2.1. To simplify analysis, we still consider that $x_{i} \in \mathbb{R}$ for all $i \in \mathcal{N}$ in this section.

First, define a matrix as

$$
\frac{\partial \Gamma(x)}{\partial x}=H(x)=\left[\begin{array}{cccc}
\frac{\partial^{2} f_{1}(x)}{\partial x_{1}^{2}} & \frac{\partial^{2} f_{1}(x)}{\partial x_{1} \partial x_{2}} & \cdots & \frac{\partial^{2} f_{1}(x)}{\partial x_{1} \partial x_{N}} \\
\frac{\partial^{2} f_{2}(x)}{\partial x_{2} \partial x_{1}} & \frac{\partial^{2} f_{2}(x)}{\partial x_{2}^{2}} & \cdots & \frac{\partial^{2} f_{2}(x)}{\partial x_{2} \partial x_{N}} \\
\vdots & \vdots & \ddots & \vdots \\
\frac{\partial^{2} f_{N}(x)}{\partial x_{N} \partial x_{1}} & \frac{\partial^{2} f_{N}(x)}{\partial x_{N} \partial x_{2}} & \cdots & \frac{\partial^{2} f_{N}(x)}{\partial x_{N}^{2}}
\end{array}\right] .
$$

Assumption 4.1. There is constant $m>0$ such that the matrix $H(x)$ satisfies that $H^{T}(x)+$ $H(x) \geq 2 m I_{N}$ for any $x \in \mathbb{R}^{N}$.

Since $f_{i}(x)$ is twice continuous differentiable, combine Assumption 4.1 and Lagrange mean value theorem we can obtain that $(x-y)^{T}(\Gamma(x)-\Gamma(y)) \geq m\|x-y\|_{2}^{2}$ holds for any $x, y \in \mathbb{R}^{N}$ [12], which means that Nash equilibrium of non-cooperative game is unique [13]. Assumption 4.1 provides a sufficient condition to guarantee the strong monotonicity of the mapping $\Gamma(x)$. Similar to the strong convexity requirement of objective function in the optimization algorithms [6], the strong monotonicity of mapping $\Gamma(x)$ plays an important role in the analysis of the convergence of the Nash equilibrium seeking algorithms.

In order to address finite-time Nash equilibrium seeking problem, the following distributed Nash equilibrium seeking algorithm is proposed:

$$
\begin{aligned}
& \dot{x}_{i}=-P_{u_{i}}^{\bar{u}_{i}}\left(\frac{\partial f_{i}\left(y_{i}\right)}{\partial x_{i}}\right), \\
& \dot{y}_{i j}=-\delta_{i} \operatorname{sign}\left(\sum_{k=1}^{N} a_{i k}\left(y_{i j}-y_{k j}\right)+a_{i j}\left(y_{i j}-x_{j}\right)\right),
\end{aligned}
$$


where $\delta_{i}>0$ and $\bar{u}_{i} \geq u_{i}>0, \forall i \in \mathcal{N}$ and $P_{u_{i}}^{\bar{u}_{i}}(\cdot)$ is defined as

$$
P_{u_{i}}^{\bar{u}_{i}}(v)=\operatorname{sign}(v) \begin{cases}\bar{u}_{i,} & |v|>\bar{u}_{i}, \\ |v|, & u_{i} \leq|v| \leq \bar{u}_{i}, \\ u_{i}, & |v|<u_{i} .\end{cases}
$$

The main idea of our proposed algorithm is that the update rate of players' actions is not infinite fast to ensure that the estimates can track other players' real actions, and the update rate of players' actions is not infinite slow to guarantee that players' actions converge to Nash equilibrium in finite time. Therefore, we designed a function $P_{u_{i}}^{\bar{u}_{i}}(\cdot)$ to limit the actions' update rate.

Theorem 4.1. Suppose that Assumptions 2.1, 2.2, 4.1 are satisfied, then the players can learn the unique Nash equilibrium in finite time under distributed algorithm (4.2).

Proof. Obviously, $\left\|\dot{x}_{i}\right\|_{2} \leq \bar{u}_{i}$ and $\|\dot{x}\|_{2} \leq \sum_{i}^{N} \bar{u}_{i}$, It follows from Theorem 3.1 that there is a finite time $T_{1}>0$ such that $y_{i j}=x_{j}$ when $t \geq T_{1}$ for all $i, j \in \mathcal{N}$ by choosing $\delta_{i}$ satisfying $\min _{i \in \mathcal{N}}\left\{\delta_{i}\right\}>\sqrt{N} \sum_{i}^{N} \bar{u}_{i}$. As mentioned in Remark 3.2 that $\min _{i \in \mathcal{N}}\left\{\delta_{i}\right\}>\sqrt{N} \sum_{i}^{N} \bar{u}_{i}$ is a sufficient condition but not necessary condition.

Next, we prove that the actions of players can converge to the unique Nash equilibrium in finite time under the proposed algorithm (4.2).

Motivated by [12], the following candidate Lyapunov function is constructed:

$$
V_{2}=\sum_{i=1}^{N} \int_{0}^{\frac{\partial f_{i}(x)}{\partial x_{i}}} P_{u_{i}}^{\bar{u}_{i}}(t) d t
$$

We call this Lyapunov function a reasonable construct because $P_{u_{i}}^{\bar{u}_{i}}(\cdot)$ is integrable and it follows from $(x-y)^{T}(\Gamma(x)-\Gamma(y)) \geq m\|x-y\|_{2}^{2}$ and $\Gamma\left(x^{*}\right)=0_{N}$ that $\|\Gamma(x)\|_{2} \geq m \| x-$ $x^{*} \|_{2}, \forall x \in \mathbb{R}^{N}$, which indicates that $V_{2}=0$ if and only if $\Gamma(x)=0_{N}$, i.e., $x=x^{*}$.

On the other hand, since

$$
\dot{x}_{i}=-P_{u_{i}}^{\bar{u}_{i}}\left(\frac{\partial f_{i}(x)}{\partial x_{i}}\right), \quad \forall t \geq T_{1}
$$

hence, for $t \geq T_{1}$, we have

$$
\begin{aligned}
\dot{V}_{2} & =\sum_{i=1}^{N} P_{u_{i}}^{\bar{u}_{i}}\left(\frac{\partial f_{i}(x)}{\partial x_{i}}\right)^{T}\left[\frac{\partial}{\partial x}\left(\frac{\partial f_{i}(x)}{\partial x_{i}}\right)\right]^{T} \dot{x} \\
& =-\left[P_{u_{i}}^{\bar{u}_{i}}\left(\frac{\partial f_{i}(x)}{\partial x_{i}}\right)\right]_{\mathrm{vec}}^{T} H(x)\left[P_{u_{i}}^{\bar{u}_{i}}\left(\frac{\partial f_{i}(x)}{\partial x_{i}}\right)\right]_{\mathrm{vec}} \\
& \leq-m\left\|\left[P_{u_{i}}^{\bar{u}_{i}}\left(\frac{\partial f_{i}(x)}{\partial x_{i}}\right)\right]_{\mathrm{vec}}\right\|_{2}^{2} \cdot
\end{aligned}
$$


Obviously,

$$
\left\|\left[P_{u_{i}}^{\bar{u}_{i}}\left(\frac{\partial f_{i}(x)}{\partial x_{i}}\right)\right]_{\mathrm{vec}}\right\|_{2}=0 \text { if and only if }\|\Gamma(x)\|_{2}=0,
$$

i.e., $V_{2}=0$. Furthermore,

$$
\left\|\left[P_{u_{i}}^{\bar{u}_{i}}\left(\frac{\partial f_{i}(x)}{\partial x_{i}}\right)\right]_{\text {vec }}\right\|_{2} \neq 0
$$

implies that there exists at lest one player $i \in \mathcal{N}$ satisfying $\frac{\partial f_{i}(x)}{\partial x_{i}} \neq 0$. According to the definition of $P_{u_{i}}^{\bar{u}_{i}}(\cdot)$, one knows that

$$
\left|P_{u_{i}}^{\bar{u}_{i}}\left(\frac{\partial f_{i}(x)}{\partial x_{i}}\right)\right| \geq u_{i} \quad \text { if } \quad \frac{\partial f_{i}(x)}{\partial x_{i}} \neq 0 .
$$

Therefore, one can obtain that if

$$
\left\|\left[P_{u_{i}}^{\bar{u}_{i}}\left(\frac{\partial f_{i}(x)}{\partial x_{i}}\right)\right]_{\mathrm{vec}}\right\|_{2} \neq 0
$$

then

$$
\min _{i \in \mathcal{N}}\left\{u_{i}^{2}\right\} \leq\left\|\left[P_{u_{i}}^{\bar{u}_{i}}\left(\frac{\partial f_{i}(x)}{\partial x_{i}}\right)\right]_{\text {vec }}\right\|_{2}^{2}
$$

and

$$
\dot{V}_{2} \leq-m \min _{i \in \mathcal{N}}\left\{u_{i}^{2}\right\},
$$

which means that $V_{2}$ decays to 0 in finite time, that is to say, $x$ converges to Nash equilibrium $x^{*}$ in finite time.

\section{Simulation examples}

Consider a non-cooperative game with 3 players and their cost functions are respectively as the following:

$$
\begin{aligned}
& f_{1}(x)=\frac{1}{2}\left(5 x_{1}^{2}+2 x_{1} x_{2}\right)+4 x_{1}, \\
& f_{2}(x)=\frac{1}{2}\left(2 x_{2} x_{1}+3 x_{2}^{2}+2 x_{2} x_{3}\right), \\
& f_{3}(x)=\frac{1}{2}\left(2 x_{3} x_{2}+4 x_{3}^{2}\right)+7 x_{3} .
\end{aligned}
$$

Take the first partial derivative of $f_{i}(x)$ with respect to $x_{i}$, we have

$$
\Gamma(x)=\left[\begin{array}{lll}
5 & 1 & 0 \\
1 & 3 & 1 \\
0 & 1 & 4
\end{array}\right]\left[\begin{array}{l}
x_{1} \\
x_{2} \\
x_{3}
\end{array}\right]+\left[\begin{array}{l}
4 \\
0 \\
7
\end{array}\right],
$$




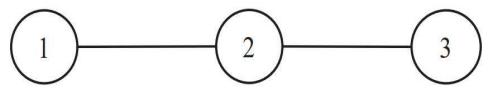

Figure 1: Communication topology of players.

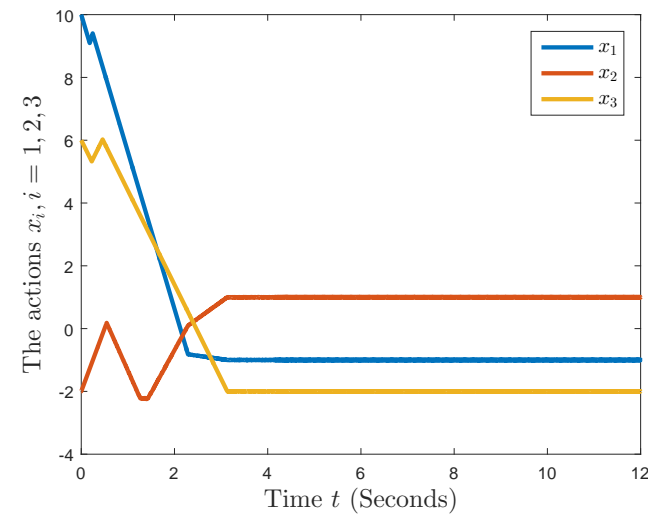

Figure 2: The actions of players under algorithm (3.3).

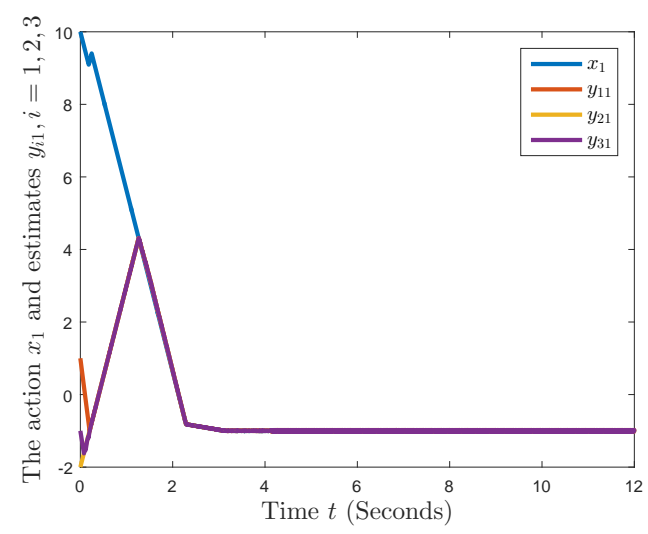

Figure 4: The estimates on $x_{1}$ of players under algorithm (3.3).

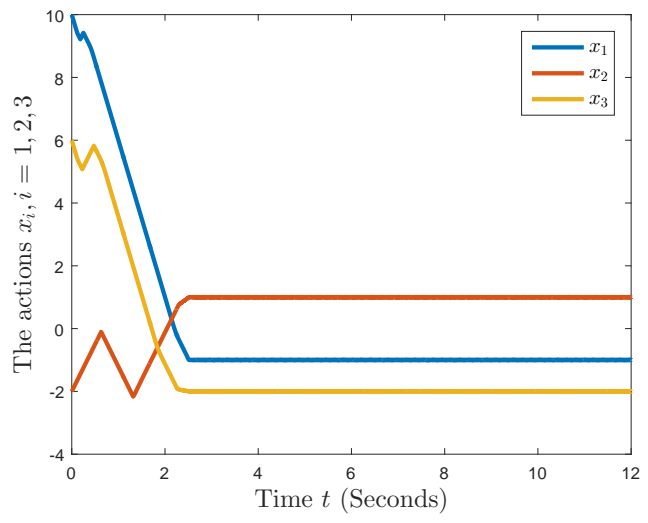

Figure 3: The actions of players under algorithm (4.2).

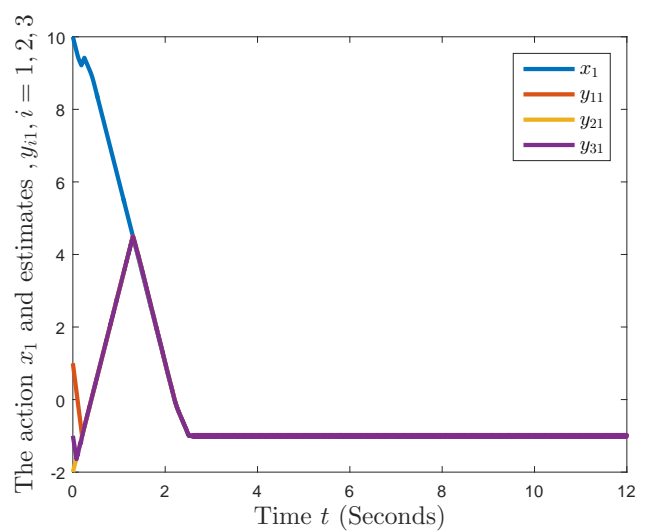

Figure 5: The estimates on $x_{1}$ of players under algorithm (4.2).

by simple calculation, we get the unique Nash equilibrium $x^{*}=[-1,1,-2]^{T}$.

Next, we verify the effectiveness of the proposed two Nash equilibrium seeking algorithms. For algorithm (3.3), take $\left[\alpha_{1}, \alpha_{2}, \alpha_{3}\right]=[5,4,3]$. For algorithm (4.2), take $\left[\bar{u}_{i}, u_{i}\right]=[5,3]$ for $i=1,2,3$. And take $\left[\delta_{1}, \delta_{2}, \delta_{3}\right]=[10,5,8], x(0)=[10,-2,6]$ and $y(0)=[1,2,3,-2,2,-4,-1,2,0]$ for both of these two algorithms. The players' communication topology is shown as Fig. 1. Fig. 2 and Fig. 3 show that the evolution of 3 players' actions under Nash equilibrium seeking algorithms (3.3) and (4.2), respectively. In order to verify that the estimates can converge to the players actions, Fig. 4 and Fig. 5 
show that the evolution of players' estimates on $x_{1}$ under Nash equilibrium seeking algorithms (3.3) and (4.2), respectively. From Figs. 2-5, one can see that the estimates converge to $x_{1}$ in two seconds and $x$ converges to $x^{*}$ in four seconds under both algorithms (3.3) and (4.2).

\section{Conclusion}

In this paper, we investigated finite-time Nash equilibrium seeking problem and designed distributed algorithms for quadratic and general non-cooperative games. It was proved that players' estimates could track the real actions in finite time by choosing appropriated parameters and the actions of players converged to Nash equilibrium in finite time under proposed algorithm. From the asymptotic convergence to the finite time convergence, it may be enlightening to accelerate the Nash equilibrium seeking. The future directions are to study how to further improve the convergence speed of Nash equilibrium seeking algorithm on the basis of guaranteed finite time convergence and consider finite-time distributed generalized Nash equilibrium seeking problem.

\section{References}

[1] T. Basar and G. Olsder, Dynamic Noncooperative Game Theory: Second Edition (Classics in Applied Mathematics). Philadelphia, PA, USA: SIAM, 1999.

[2] F. Salehisadaghiani and L. Pavel, Distributed Nash equilibrium seeking: A gossip-based algorithm, Automatica, vol. 72, pp. 209-216, 2016.

[3] D. Gadjov and L. Pavel, A passivity-based approach to Nash equilibrium seeking over networks, IEEE Trans. Autom. Control, vol. 64, no. 3, pp. 1077-1092, 2019.

[4] M. Ye and G. Hu, Distributed Nash equilibrium seeking by a consensus based approach, IEEE Trans. Autom. Control, vol. 62, no. 9, pp. 4811-4818, 2017.

[5] J. Wang and N. Elia, Control approach to distributed optimization. In Proceedings of the 48th annual Allerton conference on communication control, and computing (Allerton), pp. 557-561, 2010.

[6] S.S. Kia, J. Cortés, and S. Martínez, Distributed convex optimization via continuous-time coordination algorithms with discrete-time communication, Automatica, vol. 55, pp. 254-264, 2015.

[7] P. Lin, W. Ren, and J.A. Farrell, Distributed continuous-time optimization: nonuniform gradient gains, finite-time convergence, and convex constraint set, IEEE Trans. Autom. Control, vol. 62, no. 5, pp. 2239-2253, 2017.

[8] Z. Feng and G. Hu, Finite-time distributed optimization with quadratic objective functions under uncertain information, 2017 IEEE 56th Annual Conference on Decision and Control (CDC), pp. 208-213, 2017.

[9] Y. Song and W. Chen, Finite-time convergent distributed consensus optimisation over networks, IET Control Theory Appl., vol.10, no. 11, pp. 1314-1318, 2016.

[10] T. Tatarenko, W. Shi, and A. Nedić, Accelerated gradient play algorithm for distributed Nash equilibrium seeking, 2018 IEEE Conference on Decision and Control (CDC), pp. 3561-3566,2018, 
[11] F. Salehisadaghiani, W. Shi, and L. Pavel, Distributed Nash equilibrium seeking under partial-decision information via the alternating direction method of multipliers, Automatica, vol. 103, pp. 27-35, 2019.

[12] M. Ye, Distributed strategy design for solving games in systems with bounded control inputs, 2019 IEEE 15th International Conference on Control and Automation (ICCA), pp. 266-271, 2019.

[13] J. Rosen, Existence and uniqueness of equilibrium points for concave N-person games, Econometrica, vol. 33, no. 3, pp. 520-534, 1965.

[14] M.S. Stanković, K.H. Johansson, and D.M. Stipanović, Distributed seeking of Nash equilibria with applications to mobile sensor networks, IEEE Trans. Autom. Control, vol. 57, no. 4, pp. 904-919, 2012.

[15] S. Wen, X. He, and T. Huang, Distributed neuro-dynamic algorithm for price-based game in energy consumption system, Neural Process Lett., vol. 51, no. 1, pp. 559-575, 2019.

[16] B. Gao and L. Pavel, Continuous-time convergence rates in potential and monotone games, [Online]. Available: https://arxiv.org/abs/2011.10682 